\title{
Composição em tamanho dos peixes (Actinopterygii, Teleostei) de ribeirões da bacia do rio Tibagi, Paraná, Brasil
}

\author{
Oscar A. Shibatta \& Carolina C. Cheida
}

Museu de Zoologia, Departamento de Biologia Animal e Vegetal, Centro de Ciências Biológicas, Universidade Estadual de Londrina.86051-990 Londrina, Paraná, Brasil. E-mail: shibatta@uel.br

\begin{abstract}
Composition in size of fishes (Actinopterygii, Teleostei) from streams of rio Tibagi basin, Paraná, Brazil. The standard length of 7610 specimens of 43 species from eight streams of rio Tibagi basin was obtained in order to analyze the means of fish sizes. The higher mean size was observed in Gymnotus carapo Linnaeus, $1758(165,5 \mathrm{~mm})$ and the smaller, in Phalloceros caudimaculatus (Hensel, 1868) $(16,25 \mathrm{~mm})$. Some fish like Acestrorhynchus lacustris (Reinhardt, 1874), Leporinus obtusidens (Valenciennes, 1836), Gymnotus carapo Linneus, 1758 and Synbranchus marmoratus Bloch, 1795 can reach big or medium size when adults but were collected only young, evidencing they use some streams in beginning of life. The mean size of total species was $49,81 \mathrm{~mm}$, what shows the small size composition of fishes in the streams. The Kolmogov-Smirnov test comparing the mean size of all streams showed significant similar size both in upstream and downstream regions, independent to the different environmental conditions.
\end{abstract}

KEY WORDS. Body size, conservation, ecomorphology, streams.

A região neotropical é a mais rica em número de espécies de peixes, com estimativas de atingir até 8000 apenas em água doce (Schaefer 1998). A maior parte dessa diversidade pode ser atribuída aos peixes de pequeno a médio porte, como constatado por Vazzoler (1996), que observou 70,6\% da fauna de teleósteos do alto rio Paraná formada por espécies destas categorias de tamanho. As espécies de pequeno porte estão principalmente distribuídas em riachos, onde chegam a compor, no mínimo, 50\% da assembléia (CASTro 1999).

Segundo Vazzoler (1996) incluem-se na categoria "pequeno porte" aquelas espécies com comprimento total máximo menor que $200 \mathrm{~mm}$, "médio porte" aquelas entre 200 e 400 $\mathrm{mm}$, e "grande porte" aquelas maiores que $400 \mathrm{~mm}$. Entretanto, não há consenso quanto ao limite de tamanho de um peixe de pequeno porte, pois CASTRO (1999) atribui o comprimento igual ou inferior a $150 \mathrm{~mm}$ como limite máximo. O limite mínimo foi atribuído para peixes com comprimento total de $32 \mathrm{~mm}$, que passam a ser considerados miniaturas (WeitzMAnN 1997).

Apenas recentemente foram realizadas coletas de peixes em ribeirões da bacia do rio Tibagi, com o intuito de levantar os seus padrões de composição em espécies. Das 110 espécies coletadas, $23,6 \%$ foram consideradas exclusivas dos afluentes e subafluentes do rio Tibagi e todas eram de pequeno a médio tamanho (Shibatтa et al. 2002). Entretanto, nenhuma investigação mais profunda foi feita sobre a composição em tamanho desses animais. Desta forma, peixes de oito ribeirões da região média e baixa da bacia foram estudados, com o intuito de analisar as médias de seus tamanhos e os padrões desta característica.

\section{MATERIAL E MÉTODOS}

\section{Descrição da área de estudos}

O rio Tibagi pertence à bacia do alto rio Paraná e é o principal afluente do rio Paranapanema. Possui cerca de $550 \mathrm{~km}$ de extensão e corre inteiramente no estado do Paraná. É rico em tributários e compõe uma bacia hidrográfica com 65 afluentes principais e centenas de subafluentes (MАACK 1981).

Pode-se encontrar três designações para os pequenos rios na bacia do rio Tibagi: arroios, córregos e ribeirões (e.g. IBGE 1967). Geralmente, córregos são os cursos de água que deságuam nos ribeirões ou arroios; estes por sua vez, deságuam em outros ribeirões ou em rios. Entretanto, o termo ribeirão foi generalizado para todos os pequenos cursos de água, com largura inferior a $10 \mathrm{~m}$, inclusive trechos de cabeceiras dos rios. O termo riacho não foi utilizado por não ser mencionado na bacia. A divisão da bacia em regiões média e baixa seguiu STIPP (2000) e as localidades de coletas estão indicadas a seguir: 1) Baixo Tibagi: a) Município de Londrina: cabeceira do córrego Marrequinhas [233' $43^{\prime \prime} \mathrm{S}$ $51^{\circ} 04^{\prime} 18^{\prime \prime} \mathrm{W}$, volume médio da água no trecho de coleta $(\mathrm{Vm})=$ $17,10 \mathrm{~m}^{3}$; b) Município de Jataizinho: baixo córrego Taquari $\left(23^{\circ} 12^{\prime} 24^{\prime \prime} \mathrm{S} 50^{\circ} 56^{\prime} 50^{\prime \prime} \mathrm{W}, \mathrm{Vm}=48,23 \mathrm{~m}^{3}\right)$, alto córrego Água da Floresta $\left(23^{\circ} 12^{\prime} 24^{\prime \prime} \mathrm{S} 50^{\circ} 56^{\prime} 50^{\prime \prime} \mathrm{W}, \mathrm{Vm}=33,66 \mathrm{~m}^{3}\right)$ e médio 
ribeirão Couro do Boi $\left(23^{\circ} 17^{\prime} 09^{\prime \prime} \mathrm{S} 50^{\circ} 54^{\prime} 02^{\prime \prime} \mathrm{W}, \mathrm{Vm}=18,25 \mathrm{~m}^{3}\right)$; 2) Médio Tibagi: a) Município de Sapopema: médio ribeirão Lageadinho $\left(23^{\circ} 55^{\prime} 29^{\prime \prime} \mathrm{S} 50^{\circ} 37^{\prime} 27^{\prime \prime} \mathrm{W}, \mathrm{Vm}=22,03 \mathrm{~m}^{3}\right)$, baixo ribeirão Mambuquinhas (23⒌ $56^{\prime} 19^{\prime \prime} \mathrm{S} 50^{\circ} 43^{\prime} 42^{\prime \prime} \mathrm{W}, \mathrm{Vm}=14,98$ $\left.\mathrm{m}^{3}\right)$ e médio ribeirão Água do Luz ( $23^{\circ} 55^{\prime} 24^{\prime \prime} \mathrm{S} 50^{\circ} 37^{\prime} 51^{\prime \prime} \mathrm{W}, \mathrm{Vm}$ $\left.=41,58 \mathrm{~m}^{3}\right)$; b) Município de Mauá da Serra: cabeceira do rio Preto $\left(23^{\circ} 57^{\prime} 15^{\prime \prime} \mathrm{S} 51^{\circ} 07^{\prime} 00^{\prime \prime} \mathrm{W}, \mathrm{Vm}=42,67 \mathrm{~m}^{3}\right)$.

\section{Metodologia}

Foram realizadas coletas sazonais durante o período de março de 1999 a março de 2000 . Um trecho de $20 \mathrm{~m}$ na cabeceira ou no curso médio do ribeirão foi cercado com redes de tela fina à jusante e à montante, e as coletas foram feitas utilizando-se tarrafas, peneiras, redes de espera e arrastões. Foram feitas cinco medidas de largura e 10 de profundidade, cujas médias foram utilizadas para o cálculo do volume de água de cada trecho. Os exemplares foram fixados em formol 10\%, para posterior identificação e medições do comprimento padrão (em $\mathrm{mm})$, exceto Gymnotus carapo Linneus, 1758 e Synbranchus marmoratus Bloch, 1795 que tiveram medidos os seus comprimentos totais (CT). Foi adotado o limite máximo de $150 \mathrm{~mm}$ de comprimento padrão para a categoria de tamanho "pequeno". Posteriormente os peixes foram preservados em álcool etílico 70\% e depositados na coleção do Museu de Zoologia da Universidade Estadual de Londrina. As espécies foram identificadas utilizando obras de descrição e identificação de peixes da região neotropical, em especial para o alto rio Paraná, conforme listado em SHibatTa et al. (2002).

A comparação das médias de tamanho dos peixes das localidades foi feita com o método de Kolmogov-Smirnov, de acordo com os procedimentos apresentados por SIEGEL (1975). Para o cálculo da constância de ocorrência (C) de cada espécie nos ribeirões, foi utilizada a fórmula $C=(n / N) * 100$, onde " $n$ " é o número de ribeirões onde a espécie foi coletada e "N" é o número total de ribeirões amostrados. Quando o valor de $\mathrm{C}$ foi maior que $50 \%$, a espécie foi considerada constante, quando entre $25 \%$ e $50 \%$, a espécie foi considerada acessória, e acidental quando o valor foi menor que 25\% (DAJOZ 1973).

\section{RESULTADOS E DISCUSSÃO}

Foram coletadas 43 espécies de peixes, sendo 22 de Characiformes, 14 de Siluriformes, uma de Gymnotiformes, duas de Cyprinodontiformes, três de Perciformes e uma de Synbranchiformes (Tab. I). A dominância de Ostariophysi, principalmente de Characiformes e Siluriformes, é comum aos rios neotropicais (LOWE-MCCONNELL 1999).

Nove espécies foram constantes em mais de $60 \%$ dos ribeirões, e somaram juntas 3.462 exemplares, o que representou $45,5 \%$ do total capturado (7.610 exemplares). As espécies constantes foram Astyanax eigenmanniorum (Cope, 1894) (75\%), Astyanax sp. (75\%), Bryconamericus iheringii (100\%), Characidium aff. zebra Eigenmann, 1909 (62,5\%), Geophagus brasiliensis Kner, 1865 (62,5\%), Hypostomus ancistroides (Ihering, 1911) (87,5\%), Imparfinis schubarti (Gomes, 1956) (62,5\%), Oligosarcus paranensis Menezes \& Géry, 1983 (62,5\%) e Rhamdia quelen Quoy \& Gaimard, 1824 (87,5\%). Destas, apenas Imparfinis schubarti tinha sido coletada em ribeirões e não no rio Tibagi (Shibatta et al. 2002).

Outras espécies, consideradas acessórias, foram: Acestrorhynchus lacustris (25\%), Apareiodon ibitiensis Amaral-Campos,
1944 (50\%), Astyanax altiparanae Garutti \& Britski, 2000 (25\%), Astyanax scabripinnis (Jenyns, 1842) (25\%), Bryconamericus exodon Eigenmann, 1907 (25\%), Bryconamericus stramineus Eigenmann, 1908 (25\%), Corydoras aeneus (Gill, 1858) (25\%), Corydoras paleatus (Jenyns, 1842) (25\%), Cyphocharax modestus (Fernández-Yépez, 1948) (25\%), Hoplias malabaricus (Bloch, 1794) (25\%), Hypostomus regani (Ihering, 1905) (25\%), Odontostilbe stenodon (Eigenmann, 1905) (37,5\%), Phalloceros caudimaculatus (Hensel, 1868) (50\%), Pimelodella avanhandavae Eigenmann, 1917 (37,5\%), Serrapinnus notomelas (Eigenmann, 1915) (37,5\%), Steindachnerina insculpta (Fernández-Yépez, 1948) (25\%), Synbranchus marmoratus (25\%) e Trychomycterus sp. 2 (37,5\%). De acordo com BRITSKI (1972), a espécie Astyanax scabripinnis vive somente nas cabeceiras dos rios o que nos permite supor que ela seria considerada constante se todas as coletas fossem feitas nas regiões de cabeceiras dos ribeirões. Além desta espécie, Trichomycterus sp.2 e Phalloceros caudimaculatus foram coletadas apenas em cebeceiras dos ribeirões na bacia do rio Tibagi (ShibatTa et al. 2002).

As outras 15 espécies coletadas (Tab. I) estiveram presentes apenas em uma localidade (constância igual a 12,5\%), e foram consideradas acidentais. Aqui cabe a ressalva de que estas informações são suficientes para levantar os padrões gerais das espécies nos ribeirões do médio e baixo rio Tibagi, não servindo, entretanto, para uma análise mais detalhada de cada local, em que certamente algumas espécies mudarão de status.

Gymnotus carapo foi a espécie de maior comprimento padrão médio (165,5 mm) e Phalloceros caudimaculatus (16,25 mm), a de menor (Tab. I). Apesar desta espécie ter apresentado o menor tamanho, não pode ser categorizada como "miniatura" porque podem ultrapassar os $32 \mathrm{~mm}$. Do total de espécies, 98,3\% estiveram nas categorias entre 15 e $150 \mathrm{~mm}$ de comprimento padrão, com modas entre 25,1 a $40 \mathrm{~mm}$, e nenhuma atingindo os $200 \mathrm{~mm}$ (Fig. 1). O comprimento padrão médio das espécies constantes foi de $41,25 \mathrm{~mm}$ e o comprimento padrão médio do total das espécies foi de $49,81 \mathrm{~mm}$, permitindo assim a classificação das assembléias como de pequeno porte.

Algumas destas espécies crescem muito mais do que o observado neste trabalho (Tab. I) como, por exemplo, Acestrorhynchus lacustris, Leporinus obtusidens, Gymnotus carapo e Synbranchus marmoratus $[\mathrm{CP}=270 \mathrm{~mm}$ (Menezes 1969); $\mathrm{CP}=$ 500 mm (J.C. Garavello 1979, dados não publicados); CP = 400 mm (BRITSKi et al. 1986); CT $=1000 \mathrm{~mm}$ (BRITSKI et al. 1999); respectivamente]. Entretanto, como foram capturadas ainda muito jovens, evidenciam que os ribeirões são importantes no início da vida dessas espécies.

Segundo TокеSH (1999), o tamanho diferenciado do corpo ou de parte dele pode ser o mantenedor da coexistência das espécies pela partição de recursos e pode ser considerado como um dos fatores que definem o nicho das espécies. Entretanto, esse não deve ser o fator exclusivo, como observado no ribeirão Marrequinhas, onde outras espécies do mesmo gênero, com tamanhos semelhantes ocorreram juntas. A ecomorfologia talvez possa explicar melhor a coexistência dessas espécies. A espécie $B$. exodon pode ter distribuição restrita devido ao seu hábito alimentar, pois a boca inferior denota especialização para captura de alimento no substrato. Já a espécie $B$. iheringii possui uma forma intermediária não sendo muito alongada como B. stramineus e tendo uma boca mais terminal que a de B. exodon, o que facilita a exploração de recursos em diferentes ambientes. Além disso, a presença de B. stramineus neste local 


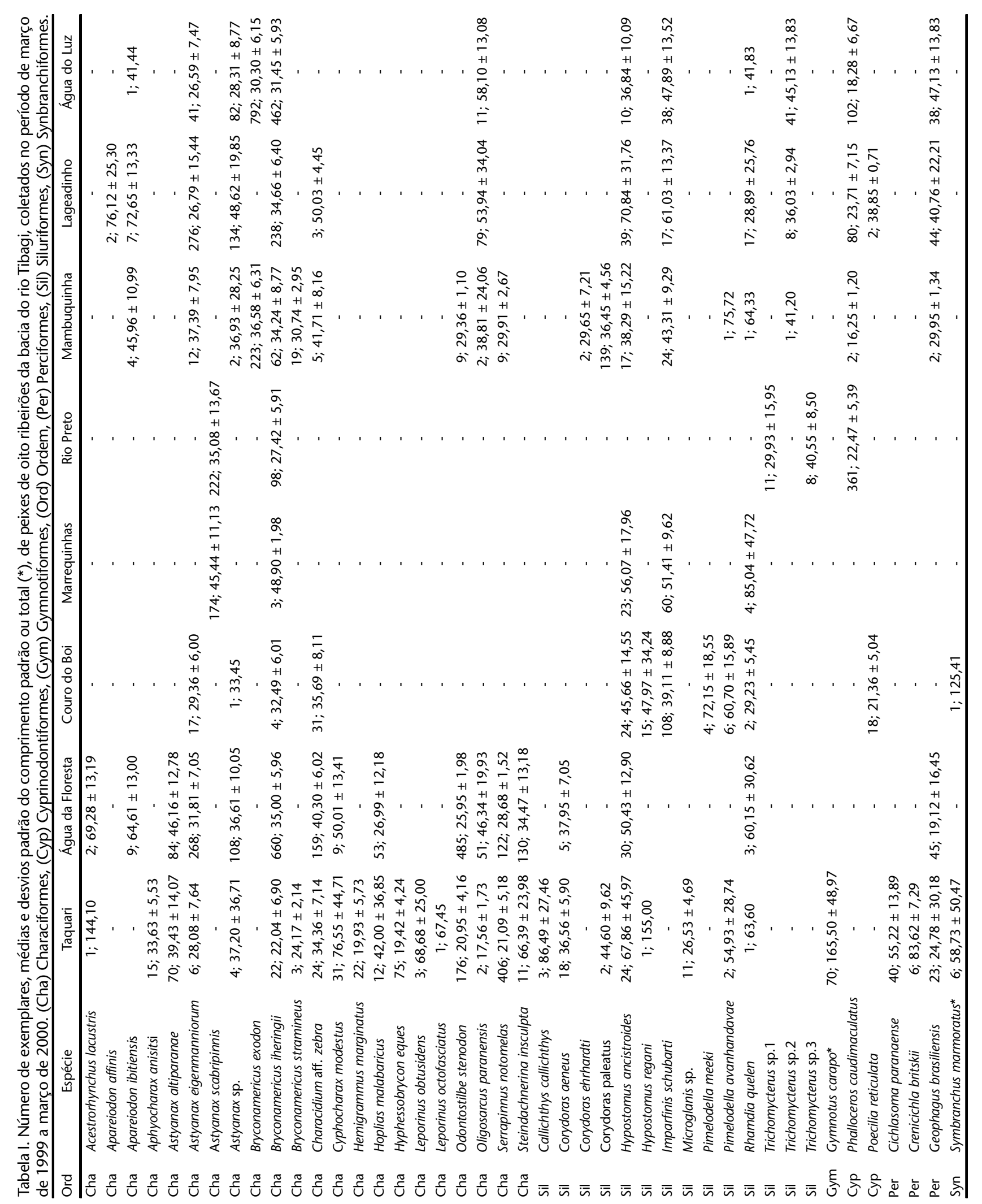

Revista Brasileira de Zoologia 20 (3): 469-473, setembro 2003 

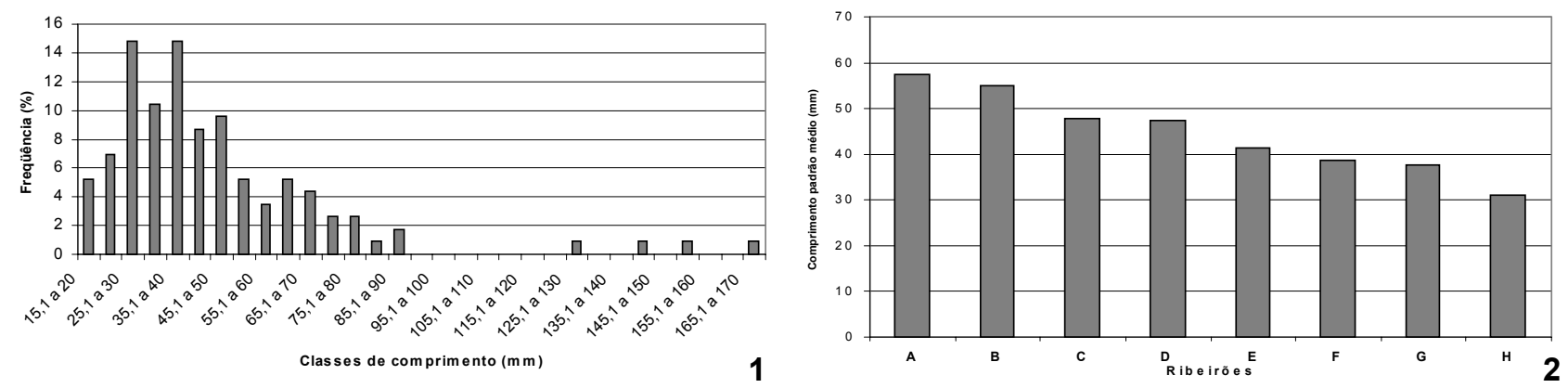

Figuras 1-2. (1) Freqüência das classes de comprimentos padrão médios ( $\mathrm{mm}$ ) dos peixes e (2) comprimento padrão médio (mm) do total das espécies de peixes coletados sazonalmente no período de março de 1999 a março de 2000, em oito ribeirões da bacia do rio Tibagi. (A) Marrequinha, (B) Taquari, (C) Couro do Boi, (D) Lageadinho, (E) Água da Floresta, (F) Mambuquinha, (G) Água do Luz, (H) Rio Preto.

pode ser explicada pela sua proximidade com o rio Tibagi, onde esta espécie ocorre com freqüência (SнiваттA et al. 2002), favorecendo a sua migração para o ribeirão em uma determinada época do ano (maior freqüência em janeiro).

Cada trecho amostrado possui uma combinação de espécies única, embora nenhum possa ser considerado como uma área de endemismo. Inclusive, pode-se verificar na literatura (GARUTTI 1988, BENNEMANn et al. 1995) que a diversidade aumenta da cabeceira para a foz em um mesmo rio. Tal fato é de importância para a conservação dos ambientes, evidenciando que não se deve generalizar as características bióticas de um local para outro. Entretanto, pouco se conhece a respeito da composição em tamanho em diferentes trechos ou diferentes condições ambientais. Dentre os ribeirões analisados, a cabeceira do ribeirão Marrequinhas e o baixo ribeirão Taquari, foram os que apresentaram a maior média do comprimento padrão dos peixes $(57,37 \mathrm{~mm}$ e $55,05 \mathrm{~mm}$ respectivamente, figura 2$)$, mas com valores muito próximos aos dos outros ribeirões. A correlação entre as médias de tamanho dos peixes de todos os ribeirões com os respectivos volumes médios da água dos trechos amostrados foi muito baixa $(r=-0,12)$, indicando que, apesar do volume de água variar, o tamanho dos peixes é muito semelhante. A mesma conclusão pode ser feita a partir do teste de Kolmogorov-Smirnov, utilizada para as médias dos comprimentos padrões entre as localidades $(\mathrm{N}=388,1 ; \mathrm{D}=0,07$; ou seja, o $p$ associado ao valor de D é maior do que 0,01$)$. Desta forma, peixes de cabeceira são significativamente do mesmo tamanho que aqueles dos trechos médio e baixo dos ribeirões.

Especula-se que um dos fatores que pode influenciar na composição em tamanho das espécies é a instabilidade das condições ambientais dos ribeirões. Segundo CASTRo (1999) o ambiente instável favoreceria linhagens de peixes pequenos com características progenéticas e com curto ciclo de vida. A redução de caracteres pode ser facilmente observada em Serrapinnus notomelas e Odontostilbe stenodon, que apresentam linha lateral incompleta. Entretanto, a redução de caracteres não ocorreu ou não é facilmente identificada em outras espécies. Apesar da biologia reprodutiva da maioria das espécies de ribeirões ainda ser pouco conhecida, é provável que elas apresentem ciclo de vida curto, reproduzindo-se no espaço de um ano. A longevidade dos peixes na bacia do rio Tibagi, entretanto, ainda é desconhecida.
Considerações históricas também podem ser feitas, principalmente no que concerne à origem das linhagens de peixes de pequeno porte. Segundo CосквURn (1991), existe uma tendência das linhagens se iniciarem com organismos de pequeno porte e irem aumentando em tamanho ao longo do tempo evolutivo. Particularmente aos Characiformes, as filogenias apresentadas por diferentes autores (Buckup 1998, Weitzman \& MaLABARba 1998) não suportam esta hipótese, pois as linhagens basais estão representadas por famílias com espécies tanto de pequeno quanto de grande porte.

\section{AGRADECIMENTOS}

A Sirlei T. Bennemann e Ângela T. Silva-Souza pelas valiosas sugestões e discussões que enriqueceram o trabalho. A Heitor Frossard, Lucia Giuliano-Caetano, Edson Santana da Silva e Aparecido de Souza pelo auxílio nas coletas de campo. Este estudo fez parte do projeto "Aspectos da fauna e flora da bacia do rio Tibagi" e teve financiamento da FINEP (1832/95) e CNPq/RHAE (610.148/96-6). C. C. C. foi bolsista de iniciação científica do PIBIC-CNPq/UEL - 1999/2000.

\section{REFERÊNCIAS BIBLIOGRÁFICAS}

Bennemann, S. T.; Â.T. Silva-Souza \& G.R.A. Rocha. 1995. Composicion ictiofaunistica em cinco localidades de la cuenca del rio Tibagi, PR - Brasil. Interciencia, Caracas, 20 (1): 7-13.

Britski, H.A. 1972. Peixes de água doce do Estado de São Paulo - Sistemática, p. 79-108. In: Poluição e Piscicultura. São Paulo, Faculdade de Saúde Pública, Universidade de São Paulo, Instituto de Pesca, 216p.

BRITSKI, H.A.; Y. SATO \& A.B.S. Rosa. 1986. Manual de identificação de peixes da região de Três Marias (com chaves de identificação para os peixes da bacia do São Francisco). Brasília, CODEVASF, $2^{\mathrm{a}}$ ed., $115 \mathrm{p}$.

Britski, H.A.; K.Z.S. Silimon \& B.S. Lopes. 1999. Peixes do Pantanal. Brasília, Embrapa, 184p.

Buckup, P.A. 1998. Relationships of the Characidiinae and Phylogeny of Characiform fishes (Teleostei: Ostariophysi), p. 123-144. In: L.R. Malabarba; R.E. Reis; R.P. Vari; Z.M.S. LuCENA \& C.A.S. LuCENA (Eds). Phylogeny and classification of Neotropical fishes. Porto Alegre, EDIPUCRS, 603p. 
CASTRO, R.M.C. 1999. Evolução da ictiofauna de riachos sulamericanos: Padrões gerais e possíveis processos causais, p.139-155. In: E.P. Caramaschi; R. Mazzoni \& P.R. Peres-Neto (Eds). Ecologia de Peixes de Riachos. Rio de Janeiro, PPGEUFRJ, Série Oecologia Brasiliensis, vol. 6, XVI+260p.

CockBurn, A. 1991. An introduction to evolutionary Ecology. Oxford, Blackwell Science, 370p.

DAJOz, R. 1973. Ecologia geral. São Paulo, Ed. Vozes Ltda e EDUSP, 472p.

GarutTi, V. 1988. Distribuição longitudinal da ictiofauna em um córrego da região noroeste do Estado de São Paulo, bacia do rio Paraná. Revista Brasileira de Biologia, Rio de Janeiro, 48 (4): 747-759.

IBGE. 1967. Curiúva. Carta do Brasil. Rio de Janeiro, Instituto Brasileiro de Geografia, Escala 1:1.000.000, folha SG-22D-11.

Lowe-McConNell, R.H. 1999. Estudos ecológicos de comunidades de peixes tropicais. São Paulo, EDUSP, 534p.

MAACK, R. 1981. Geografia física do estado do Paraná. Rio de Janeiro, Livraria José Olympio Editora S.A., 450p.

Menezes, N.A. 1969. Systematics and evolution of the tribe Acestrorhynchini (Pisces, Characidae). Arquivos de Zoologia, São Paulo, 18 (1-2): 1-150.

SCHAEFER, S.A. 1998. Conflict and resolution: impact of new taxa on Phylogenetic studies of the Neotropical cascudinhos (Siluroidei: Loricariidae), p. 375-400. In: L.R. Malabarba; R.E.
Reis; R.P. Vari; Z.M.S. Lucena \& C.A.S. Lucena (Eds). Phylogeny and classification of Neotropical fishes. Porto Alegre, EDIPUCRS, 603p.

SIEGEL, S. 1975. Estatística não-paramétrica para as ciências do comportamento. São Paulo, McGraw-Hill, 350p.

Shibatta, O.A.; M.L. Orsi; S.T. Bennemann \& Â.T. Silva-SouZa. 2002. Diversidade e distribuição de peixes na bacia do rio Tibagi, p. 399-419. In: M.E. Medri; E. Bianchini; O.A. Shibatta \& J.A. Pimenta (Eds). A bacia do rio Tibagi. Londrina, M.E. Medri, $595 \mathrm{p}$.

STIPP, N.A.F. 2000. Macrozoneamento ambiental da bacia hidrográfica do rio Tibagi (PR). Londrina, Editora Universidade Estadual de Londrina, 196p.

TOKESHI, M. 1999. Species coexistence. Ecological and evolutionary perspectives. Oxford, Blackwell Science, 454p.

Weitzman, S.H. 1997. Comments on miniature freshwater fishes. Tropical Fish Hobbyist, Neptune City, 45 (5): 136-154.

Weitzman, S.H. \& L.R. Malabarba. 1998. Perspectives about the phylogeny and classification of the Characidae (Teleostei: Characiformes), p. 162-170. In: L.R. MALABARBA; R.E. ReIs; R.P. Vari; Z.M.S. Lucena \& C.A.S. Lucena (Eds) Phylogeny and classification of Neotropical fishes. Porto Alegre, EDIPUCRS, 603p.

VAZzoler, A.E.A.M. 1996. Biologia da reprodução de peixes teleósteos: teoria e prática. Maringá, EDUEM/SBI/CNPq/ Nupélia, 169p.

Recebido em 13.II.2003; aceito em 20.VIII.2003. 